\title{
Chemopreventive Effects of Licorice and Its Components
}

\author{
Ann M. Bode $\cdot$ Zigang Dong
}

Published online: 28 January 2015

(C) Springer International Publishing AG 2015

\begin{abstract}
Cancer is still a major health issue worldwide and identifying novel but safe compounds for prevention and treatment is a high priority. Licorice (Glycyrrhiza) is a perennial plant that is cultivated in many countries and has been reported to exert antioxidant, anti-inflammatory and anticancer effects. However, some components of licorice exert unwanted side effects and therefore identifying safer licorice components would be ideal. The anticancer activities of many of the licorice components appear to include cycle arrest, apoptosis induction, and general antioxidant effects. Commonly reported indirect protein targets important in tumorigenesis include many cell cycle-related proteins, apoptosisassociated proteins, MMP proteins, COX-2, GSK- $\beta$, Akt, NF- $\mathrm{KB}$, and MAP kinases. Importantly, several licorice components were reported to directly bind to and inhibit the activities of PI3-K, MKK4, MKK7, JNK1, mTOR, and $\mathrm{Cdk} 2$, resulting in decreased carcinogenesis in several cell and mouse models with no obvious toxicity. This review focuses on specific components of licorice for which a direct protein target has been identified.
\end{abstract}

Keywords Glycyrrhizic acid · Glycyrrhetic acid · Iso-liquiritigenin · Isoangustone A · Licochalcone A · Licochalcone $\mathrm{E}$

This article is part of the Topical Collection on Cancer Chemoprevention

A. M. Bode $(\bowtie) \cdot$ Z. Dong

The Hormel Institute University of Minnesota, 801 16th Ave NE, Austin, MN 55912, USA

e-mail: ambode@hi.umn.edu

Z. Dong

e-mail: zgdong@hi.umn.edu

\section{Introduction}

Despite efforts and advances in prevention and treatment, cancer is still a major cause of death in most countries. Cancer is a worldwide health threat with major physical, social, and economic burdens. Because cancer is believed to be preventable, attention has centered on dietary phytochemicals and natural compounds as effective interventions in cancer development. However, the failure of many large-scale clinical trials has questioned whether diet-based cancer prevention or therapy can succeed. Two major weaknesses plagued many of these trials: (1) a lack of mechanism-based preclinical studies to support the trial; and (2) a lack of knowledge regarding the specific molecular or cellular targets of the selected dietary agents or functional foods. One of the greatest challenges for researchers is to reduce the accumulation of distortion and half-truths reported in the popular media regarding the health benefits of certain foods or food supplements. The use of these substances is not new, but interest has increased dramatically because of perceived health benefits presumably acquired without unpleasant side effects. However, the effects of long-term consumption of food supplements and specific dietary factors have not been extensively studied.

Many anticancer drugs exert adverse side effects, which can be severe or deadly. Thus, identification of novel anticancer compounds from natural products was proposed as a safer alternative and a promising strategy for cancer prevention or treatment. Many traditional herbal medicines and certain food constituents exhibit anti-inflammatory and antioxidant effects, suggesting their potential as chemopreventive or therapeutic agents. Licorice (Glycyrrhiza) is a perennial plant that is cultivated in several countries, including China, Russia, Spain, Persia, and India. Licorice extract from the root is very sweet and is a common source of licorice used in traditional and herbal medicines [1]. Licorice is one of the oldest referenced botanicals with a written record referring to the use of 
licorice dated $2100 \mathrm{BC}$ [2]. Notably, about $90 \%$ of the licorice produced is used as a flavoring agent for tobacco products, such as American blend cigarettes, moist snuff, chewing tobacco, and pipe tobacco [3]. Licorice flavoring is added to candies, chewing gum, soft drinks (e.g., root beer) and other beverages (e.g., herbal teas), toothpaste, and herbal remedies for cough and stomach problems and the overconsumption of these products is associated with increased risk of licorice intoxication [4]. Licorice is consumed worldwide and estimates suggest an annual consumption of about $1.5 \mathrm{~kg} /$ person [5], with some individuals consuming as much as $100 \mathrm{~g}$ of licorice per week [6]. Glycyrrhetic acid from licorice is associated with hypertension [7]. Consuming as little as $50 \mathrm{~g}$ or $2 \mathrm{oz}$ of licorice, which contain about $75 \mathrm{mg}$ glycyrrhetic acid, daily over a 2-week-period reportedly caused a significant increase in blood pressure [8].

In contrast, evidence intimates that licorice root possesses antioxidant, anti-inflammatory, antiviral, antitumor, and other protective bioactivities [9-11]. Licorice or its extract have been used for prevention of hepatitis and allergic reactions [1], treatment of esophageal inflammation and gastric ulcer [9, 12] and as a depigmentation agent in cosmetic and pharmaceutical products [13].

Licorice root contains a variety of oils, alkaloids, polysaccharides, polyamines, triterpenes, phenolic acids, flavones, flavans, chalcones, flavonoids, and isoflavonoids [14-16]. The major component of licorice is glycyrrhizin or glycyrrhizic acid $[10,17]$ that is hydrolyzed in the intestine to the active glycyrrhetic acid (GA) by $\beta$-glucuronidase [18]. GA in licorice extracts has been associated with mineralocorticoid excess [19] and is a potent inhibitor of 11- $\beta$ hydroxysteroid dehydrogenase, which can result in increased cortisol activity [20].

The anti-inflammatory and antioxidant effects of licorice components and extracts and their potential role in numerous diseases, including cancer, have been studied extensively. However, most of the studies are observational in nature and a direct molecular target has not been unequivocally identified to explain the potential beneficial effects of licorice. In this review, we will focus not only on what is known about glycyrrhizic acid and glycyrrhetic acid (Fig. 1a, b) in cancer prevention, but also on certain specific components of licorice, which may be less toxic and for which a potential molecular anticancer target has been identified. The specific components to be reviewed include the chalcone-type derivative iso-liquiritigenin (ILQ, Fig. 1c), the flavonoid isoangustone A (IAA, Fig. 1d), and the licochalcones, especially licochalcone A (LicA; Fig. 1e) and E (LicE; Fig. 1f).

\section{Glycyrrhizin and Glycyrrhetic Acid}

A number of studies have focused on the bioactivity of glycyrrhizin (Gc) and glycyrrhetic acid (GA). Gc has been used against chronic viral hepatitis [21] and is reportedly active against a number of other viruses, including latent Kaposi sarcoma-associated herpes virus [22], severe acute respiratory distress syndrome (SARS) [23, 24], and EpsteinBarr Virus (EBV) (Lin, 2008 \#307). It was reported to exert protection against carbon tetrachloride-induced liver injury in mice [25], liver damage associated with aflatoxin-induced oxidative stress [26] and other types of liver damage induced by oxidative stress [27]. These licorice compounds also can induce interferon production $[26,28]$ and protect against neurotoxicity by modulating the binding of nuclear factorkappaB to its molecular targets [29].

Gc or GA has been reported to inhibit 7,12-dimethylbenz (a) anthracene (DMBA)-initiated skin tumor formation in mice [30]. Researchers have also shown that Gc or GA reduced melanoma cell proliferation [31] and metastasis [32]. Other cancers that have been reported to be sensitive to the antiproliferative effects of GA or Gc include UVB-induced skin carcinogenesis [33•], prostate cancer [34], stomach cancer and leukemia $[35,36]$, gastric cancer [37], endometrial carcinogenesis [38], pituitary adenomas [39], and breast cancer [40]. GA was reported to suppress A549 and NCI-H460 non-small cell lung cancer (NSCLC) growth and inhibit thromboxane synthase activity [41]. It did not affect human 16HBE-T bronchial epithelial cell growth or the growth of NCI-H23 lung cancer cells, which express low levels of thromboxane synthase activity [41]. GA also induced apoptosis and G1 arrest in NCI-H460 NSCLC cells as was evidenced by increased expression of cleaved poly (ADP-ribosyl) polymerase (PARP) and caspase 3, and attenuated expression of Bcl-XL, Bcl-2, cyclin D1, and cyclin E [42]. In addition, GA treatment corresponded with decreased phosphorylation of protein kinase $\mathrm{C}(\mathrm{PKC} \alpha, \beta)$ and extracellular activated protein kinases (ERKs) as well as increased phosphorylation of PKC $\delta$ and c-Jun NH2-terminal kinases (JNKs), which affect cell death [42].

Mechanistically, the molecular or cellular targets of licorice, Gc or GA, have only been hinted at and not specifically identified. The effects of GA were examined in an UVBirradiation-induced skin tumor study and results indicated that feeding mice GA before UVB irradiation $\left(180 \mathrm{~mJ} / \mathrm{cm}^{2}\right.$ twice per week) resulted in delayed tumor appearance and decreased multiplicity and size [29]. Mice fed GA for 2 weeks before a single UVB exposure $\left(180 \mathrm{~mJ} / \mathrm{cm}^{2}\right)$ exhibited decreased UVB-induced thymine dimer-positive cells, reduced expression of proliferative cell nuclear antigen (PCNA), and exhibited fewer TUNEL (terminal deoxynucleotidyl transferasemediated dUTP nick end labeling)-positive cells. Animals displayed increased p53- and p21/Cip1-positive cells in the epidermis. These investigators also observed inhibition of NF- $k B$ and cyclooxygenase-2 (COX-2) activities and decreased prostaglandin $\mathrm{E}_{2}\left(\mathrm{PGE}_{2}\right)$ and nitric oxide $(\mathrm{NO})$ levels, which appears to be a common observation. The evidence 
Fig. 1 Chemical structures of a glycyrrhizic acid; b glycyrrhetic acid; c iso-liquiritigenin; $\mathbf{d}$ isoangustone $\mathrm{A} ; \mathrm{e}$ licochalcone $\mathrm{A}$; f licochalcone $\mathrm{E}$

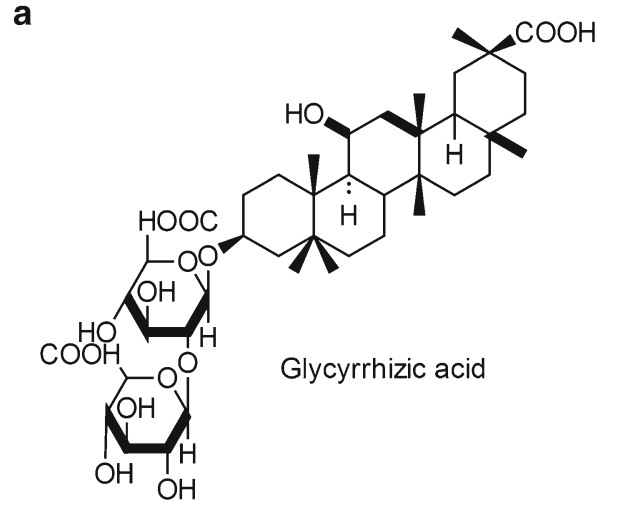

b

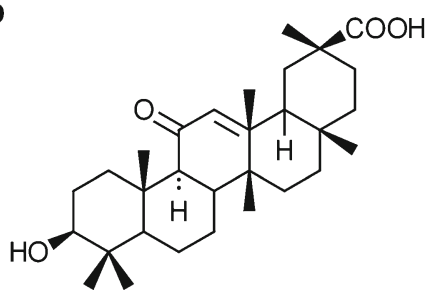

Glycyrrhetic acid<smiles>O=C(/C=C/c1ccc(O)cc1)c1ccc(O)cc1</smiles>

Iso-liquiritigenin

d<smiles>CC(C)=CCc1cc(-c2coc3cc(O)c(CC=C(C)C)c(O)c3c2=O)cc(O)c1O</smiles><smiles>C=CC(C)(C)c1cc(/C=C/C(=O)c2ccc(O)cc2)c(O)cc1OC</smiles>

Licochalcone A f<smiles>C=C(C)[C@H](C)c1cc(/C=C/C(=O)c2ccc(O)cc2)c(O)cc1O</smiles>

(+)-Licochalcone E suggested that the anticancer effects of GA were mediated through the blockade of NF-kB activation by interfering with the phosphorylation or degradation of IKB [29]. A more recent study indicated that GA supplementation suppressed the development of 1,2-dimethyhydrazine (DMH)-induced precancerous lesions in Wistar rat colon. It also reduced the infiltration of mast cells, attenuated the expression of Ki-67, NF- $\mathrm{kB} /$ p65, COX-2, inducible nitric oxide synthase (iNOS) and vascular endothelial growth factor (VEGF), while enhancing the expression of $\mathrm{p} 53$, connexin- 43 , caspase-9, and cleaved caspase-3, which suggested the induction of apoptosis [43], also agreeing with other studies [29, 42]. GA reportedly induced G0/G1 phase arrest, apoptosis, and DNA damage in WEHI-3 leukemia cells by increasing reactive oxygen species (ROS) levels and caspase-3 activity in these cells [44]. Researchers concluded that GA induced apoptosis through the death receptor, mitochondria-mediated, and endoplasmic reticulum (ER) stress signaling pathways [44].

Even though GA or Gc appears to have good anticancer effects, as indicated earlier, long-term consumption of licorice, Gc or GA, appears to cause mineralocorticoid excess, hypertension, and hypokalemia [4, 10, 45-48]. GA overdosing can also lead to fatigue, muscle weakness, cardiac arrhythmias, fluid retention, rhabdomyolosis (i.e., breakdown of muscle fibers leading to release of myoglobin into the blood, which can cause kidney damage), and myoglobinuria (i.e., myoglobin in the urine) [1, 4, 48, 49]. Based on these adverse effects, identifying other less toxic cancer preventive components of licorice is extremely important.

\section{Iso-Liquiritigenin}

Iso-liquiritigenin [(E)-1-(2,4-dihydroxyphenyl)-3-(4hydroxyphenyl) prop-2-en-1-one; ILQ; Fig. 1c] is a chalconoid compound found in licorice that has been identified as having anticancer activity. For example, dietary administration of ILQ delayed DMBA-induced mammary tumor growth in female Sprague--Dawley rats [50]. When administered in the diet to ovariectomized athymic mice transplanted with MCF-7 cells ransfected with aromatase (CYP19), ILQ inhibited growth of these breast cancer cells in vivo with no apparent toxicity, suggesting that ILQ could act as an 
aromatase inhibitor [51]. However, ILQ appears to act through several nonspecific mechanisms with modulation of matrix metallopeptidases (MMP), COX-2, and induction of apoptosis being the most widely reported.

Proteases of the MMP family are involved in extracellular matrix (ECM) breakdown in embryonic development, angiogenesis, and wound healing, but also can play a role in metastasis [52]. Dysfunction of hypoxia-inducible factor-1 (HIF-1) [53], VEGF [54], and MMP-9 and -2 proteins [55] is common in many cancers. ILQ was reported to inhibit expression of HIF-1, VEGF, and MMP-9 and -2 and reduce breast cancer cell migration [56]. These effects appeared to be at least partially associated with modulation of NF-kB, p38, and phosphatidylinositol 3-kinase (PI3-K)/protein kinase B (Akt) signaling [56]. ILQ was also able to attenuate phorbol myristate acetate (PMA)-induced expression of membrane type 1-MMP and MMP-2 in human umbilical vein endothelial cells (HUVECs) [57]. ILQ also inhibited PMA-induced migration and diminished PMA-stimulated JNKs and p38 signaling [57].

Others have reported that the effects of ILQ correlate with COX-2 expression. For example, ILQ inhibited PMA-induced COX-2 expression in non-tumorigenic MCF-10A mammary epithelial cells and the effect was associated with reduced ERKs activity [58]. ILQ decreased $\mathrm{PGE}_{2}$ and $\mathrm{NO}$ production in RAW264.7 mouse macrophage cells and the decrease corresponded with reduced expression of COX-2 and iNOS [59]. Oral administration of ILQ (3, 15 , or $75 \mathrm{mg} / \mathrm{kg}$ ) for 12 weeks significantly reduced colon cancer multiplicity, incidence, and tumor size in a mouse model of colitis-associated tumorigenesis induced by azoxymethane (AOM)/dextran sodium sulfate (DSS) [60 ${ }^{\bullet}$. Note that a combination of a single dose of AOM with subsequent exposure to the inflammatory agent DSS in rodents is an animal model that can dramatically shorten the latency time for induction of colorectal cancer and effectively reiterates the aberrant crypt foci-adenoma-carcinoma sequence that occurs in human CRC. In this study the decreased tumorigenesis corresponded with inhibition of M2 macrophage polarization, which has been implicated in colon cancer development. This effect was accompanied by downregulation of $\mathrm{PGE}_{2}$ and IL-6 signaling [60•], suggesting an involvement of the COX enzymes.

The majority of reports regarding the anticancer activity of ILQ suggest that this compound mainly induces apoptosis. Treatment of A549 NCLSC cancer cells with a combination of licorice polyphenols, including liquiritin, isoliquiritin, or isoliquirigenin, induced apoptosis that was mediated by upregulation of p53 and p21 [61]. Another report indicated that ILQ clearly attenuated mouse and human colon carcinoma cell growth and induced apoptosis in these cells [59]. Notably, oral administration of ILQ attenuated the induction of preneoplastic aberrant crypt foci in the colon of male F344 rats [59]. This licorice compound abrogated G2/M cell cycle progression and effectively induced apoptosis in HeLa human uterine cervical cancer cells [62]. Investigators observed activation of ataxia telangiectasia-mutated (ATM), increased phosphorylation of p53 (Ser15) and changes in several cell cycle proteins, including cyclin B, cyclin A, cell division cycle protein $(\mathrm{Cdc}) 2$, cdc25C, and checkpoint kinase (Chk)2. Apoptosis corresponded with changes in pro-apoptotic Bax and Bak and decreased levels of antiapoptotic Bcl-2 and Bcl- ${ }_{-\mathrm{XL}}$ in HeLa cells [62]. Interestingly, ILQ could inhibit in vivo lung metastasis of MDA-MB-231 breast cancer cells by preventing resistance to anoikis [63•], which is a type of apoptosis induced by anchorage-dependent cells detaching from the extracellular matrix around them [64]. Resistance to anoikis can occur at several stages of metastasis [65]. The inhibitory effect of ILQ on migration and invasion was mediated through a downregulation of COX-2 and cytochrome P450 4A signaling [63•]. Other observations associated with reversal of resistance to anoikis included decreased phosphorylation levels of the oncoproteins, PI3-K (Tyr458), PDK (Ser241), and Akt (Thr308) [63•].

All of these studies suggest a potent anticancer effect of ISL, but a direct molecular target was not identified. Our group investigated the effects and mechanism of ILQ on the growth of tyrosine kinase inhibitor (TKI)-sensitive and TKIresistant NSCLC cells. Treatment with ILQ inhibited growth and induced apoptosis in both cell types. ILQ caused apoptosis that correlated with the cleavage of caspase- 3 and PARP, increased expression of Bim and reduced expression of Bcl-2. ILQ inhibited the catalytic activity of both wildtype and double-mutant (L858R/T790M) epidermal growth factor receptor (EGFR). Treatment with ILQ inhibited anchorageindependent growth of NIH3T3 cells stably transfected with either wildtype or double-mutant EGFR. ILQ also reduced the phosphorylation of Akt and ERKs in both TKI-sensitive and resistant NSCLC cells, and suppressed the kinase activity of Akt1 and ERK2 in vitro. Importantly, ILQ directly interacted with both wildtype and double-mutant EGFR in an ATPcompetitive manner. A docking model study showed that ILQ formed 2 hydrogen bonds (Glu762, Met793) with wildtype EGFR and 3 hydrogen bonds (Lys745, Met793, Asp855) with mutant EGFR. Notably, ILQ attenuated the xenograft tumor growth of H1975 lung cancer cells, which was associated with decreased expression of Ki-67 and diminished phosphorylation of Akt and ERKs. This is the first report of a direct target of ILQ, which suppressed NSCLC cell growth by directly interacting with wildtype or mutant EGFR (in press JBC).

\section{Isoangustone $\mathrm{A}$}

Isoangustone A (IAA) is a novel flavonoid compound found in licorice root that has been reported to exert anticancer activity mainly by affecting cell cycle and apoptosis. 
Unrestrained growth and unregulated cell cycle are crucial characteristics of cancer cells and important for cancer progression [66]. The cell cycle is rigidly controlled by cyclin/ cyclin-dependent kinase (Cdk) complexes [67]. Cyclins D and E bind to Cdk4/6 and Cdk2, respectively, and sequentially phosphorylate the retinoblastoma protein $(\mathrm{Rb})$, which facilitates the transition from $\mathrm{G} 1$ to $\mathrm{S}$ phase [68].

IAA was reported to stimulate caspase-dependent pro-apoptotic signaling in SW 480 human colorectal adenocarcinoma cells [69]. Apoptosis appeared to be driven by a disruption of the mitochondrial outer membrane resulting in apoptosis [69]. IAA also could induce apoptosis in hormone-insensitive DU145 prostate cancer cells, which coincided with increased cleavage of PARP and caspases [70]. This same group reported that IAA decreased DNA synthesis and induced G1 phase arrest not only in DU145 cancer cells but 4T1 mouse mammary cancer cells [71]. The cell cycle arrest corresponded with decreased Cdk2 activity and reduced levels of Cdk2, Cdk4, cyclin A and cyclin D1 proteins [70]. We reported that IAA, but not Gc (up to $20 \mu \mathrm{M}$ ) inhibits the growth of the highly metastatic human PC3 prostate cancer cells and importantly, IAA ( 1 or $5 \mathrm{mg} / \mathrm{kg}$ ) effectively suppressed the growth of PC3 xenograft tumors in BALB/c nu/nu mice [72••]. The inhibitory effect corresponded with an induction of G1 phase arrest and the accumulation of the $p 27 \mathrm{kip} 1$ tumor suppressor gene $[72 \bullet \cdot]$. However, IAA had no effect on other cell cycle proteins, including p21cip1, cyclin D1, Cdk2, or Cdk4 in PC3 cells. IAA treatment also coincided with reduced phosphorylation of p27kip1 (Thr187), a post-translational modification that marks it for degradation [73], suggesting that IAA could upregulate p27kip1 in PC prostate cancer cells by blocking its phosphorylation [72••]. Pull-down assays indicated that IAA directly binds active mTOR and to the Cdk2 complex (Lee, 2013 \#8), which are associated directly or indirectly with regulation of p27kip expression [74]. Loss of p27kip1 expression in human prostate cancer correlates closely with tumor grade and is a prognostic marker for prostate cancer $[75,76]$.

Our group compared the anticancer activity of IAA and Gc against melanoma cells and found that IAA was more effective than Gc in attenuating growth of SK-MEL-2, 5, 28, and WM266-4 human melanoma cells [77]. Notably, IAA $(20 \mu \mathrm{M})$, but not Gc (up to $20 \mu \mathrm{M}$ ) could suppress the anchorageindependent growth of SK-MEL-28 melanoma cells. IAA treatment caused cell cycle arrest at G1 phase, but treatment with Gc had no effect on cell cycle [77]. Because treatment with IAA did not affect the number of cells in sub-G1, IAAinduced growth inhibition was not attributable to apoptosis.

Overexpression of the cyclin D1 gene and protein plays an important role in fueling carcinogenesis [78]. Melanoma often exhibits overexpression of the cyclin D1 and cdk4 genes, which regulate cell cycle progression [79]. The PI3-K/Akt signaling pathway is also important for cell survival and growth [80] and its overexpression or excessive activation has been observed in numerous tumor cell types [81]. Glycogen synthase kinase (GSK)-3 $\beta$ is a downstream target of PI3$\mathrm{K}$, which mediates cyclin $\mathrm{D} 1$ degradation in response to growth signals [82]. PI3-K-dependent phosphorylation of GSK-3 $\beta$ at Ser9 inhibits activation of GSK-3 $\beta$, which stabilizes cyclin D1 [82]. We reported that IAA, not Gc, decreased the abundance of G1 phase-related proteins, including cyclin D1 and cyclin E in SK-MEL-28 cells [77••]. Phosphorylation of $\mathrm{Rb}$ is crucial for $\mathrm{S}$ phase transition and is the most recognized substrate of cyclin D-dependent kinases and IAA markedly attenuated $\mathrm{Rb}$ phosphorylation at Ser807/Ser811, the known sites of $\mathrm{Rb}$ phosphorylated by $\mathrm{Cdk} 4$ [77 •*]. Further, IAA inhibited the phosphorylation of Akt (Ser473, Thr308) and GSK3 $\beta$ (Ser9), which play a role in the regulation of the degradation of cyclin D1 [82]. Notably, we also found that IAA suppresses PI3-K, MKK4, and MKK7 kinase activities by directly binding in an ATP-competitive manner. Detailed molecular binding results confirmed that IAA binds to the ATP binding pocket of each protein forming numerous important interactions with various amino acids [77••]. Most importantly, we found that IAA suppresses growth of SKMEL-28 cells by binding directly to PI3-K, MKK4, and MKK7 in SK-MEL-28 xenograft tumors [77••]. Collectively, these results indicate that IAA is a multi-kinase inhibitor that directly suppresses cell cycle progression.

\section{Licochalcones}

\section{Licochalcone A}

Licochalcone A (LicA) is a major phenolic constituent of licorice reportedly exhibiting antiproliferative and antiinflammatory properties in human and mouse cells [83]. It was reported to exert inhibitory effects against cancer and most reports suggest that, similar to the other licorice components, it induces apoptosis and cell cycle arrest. However, it also appears to exert some effects through its antioxidant activities.

\section{Apoptosis and Cell Cycle Effects}

LicA was reported to induce apoptosis of various cancer cell types. For example, LicA induced apoptosis in MCF-7 breast cancer and HL-60 human promyelocytic leukemia cell lines. Apoptosis was observed as cleavage of PARP, decreases in the antiapoptotic protein $\mathrm{Bcl}-2$, and changes in the $\mathrm{Bcl}-2 / \mathrm{Bax}$ ratio in favor of apoptosis [84]. Combining LicA with geldanamycin, an antibiotic inhibitor of heat shock protein 90, induced greater apoptosis of human epithelial ovarian carcinoma cell lines OVCAR-3 and SK-OV-3 than either compound alone [85]. Apoptosis corresponded with increases in ROS and caspase 8 and activation of pro-apoptotic Bid (BH3 interacting-domain death agonist) and subsequent 
depletion of reduced glutathione (GSH) ([85] \#10). Similarly, treatment of OVCAR-3 and SK-OV-3 ovarian cancer cells with a combination of Lic A and dydroxymethyl-2'-furyl)-1benzyl indazole (YC-1; a compound that sensitizes cancer cells to anticancer drugs) induced changes that indicated heightened apoptosis, including decreased Bcl-2 and survivin protein levels, increased Bax and p53 levels and enhanced cytochrome $\mathrm{c}$ release, and activation of caspases and PARP-1 [86]. LicA also suppressed the growth of CT-26 colon cancer implants in BALB/c mice and enhanced apoptosis [87]. It induced apoptosis of DU145 prostate cancer cells [88] and also caused caspase-dependent and autophagy-related cell death in human LNCaP prostate cancer cells [89]. Autophagy involves the catabolism of unnecessary or dysfunctional cellular components through the actions of lysosome. Treatment of cells with LicA resulted in formation of autophagic vacuoles and acidic vesicular organelles, and autophagosome membrane association of microtubule-associated protein 1 light chain 3, which are known characteristics of autophagy [89]. Autophagy induction was accompanied by downregulation of Bcl-2 and inhibition of the mTOR pathway [89]. LicA decreased the viability of KB human oral cancer cells but had no effect on primary normal human oral keratinocytes [90]. LicA appeared to act by inducing apoptosis that was mediated by the expression of factor associated suicide ligand (FasL) and activated caspases 8 and 3 and PARP [90].

LicA was also shown to cause cell cycle arrest resulting in apoptosis. It inhibited MKN-28, AGS, and MKN-45 gastric cancer cell growth in a dose-dependent manner $\left(\mathrm{IC}_{50}\right.$ $40 \mu \mathrm{M}$ ) [83] by causing cell cycle arrest at the $\mathrm{G} 2 / \mathrm{M}$ transition and inducing apoptosis, concomitant with increased expression of $\mathrm{Rb}$ and decreased expression of cyclin $\mathrm{A}$, cyclin $\mathrm{B}$, and MDM2 and increased expression of PARP and caspase-3 [83]. In another report, LicA also inhibited cell cycle progression by arresting cells in G2/M. This effect was accompanied by suppression of cyclin B1 and Cdc2 activities, decreased phosphorylation of $\mathrm{Rb}$ (Ser780) and attenuated expression of transcription factor E2F, which binds to Rb [91]. These changes occurred concurrently with reduction of cyclin D1, downregulation of CDKs 4 and 6, but increased cyclin E expression [91]. Others reported that LicA $(5 \mu \mathrm{M})$ inhibited plateletderived growth factor (PDGF)-induced rat vascular smooth muscle cell (rVSMC) proliferation, which was associated with decreases in PDGF-induced expression of cyclin A, cyclin $\mathrm{D} 1, \mathrm{CDK} 2$, and CDK4, and the phosphorylation of Rb [92]. Licochalcone A also reversed the decrease in p27kip1 expression and attenuated the PDGF-induced activation of ERKs [92].

LicA reportedly can suppress angiogenesis in cells and mice. LicA inhibited proliferation $(20 \mu \mathrm{M})$, migration (5$20 \mu \mathrm{M})$, and tube formation $(10-20 \mu \mathrm{M})$ of HUVECs and microvessel growth $(10-20 \mu \mathrm{M})$ [87]. These effects corresponded with downregulation of VEGF receptor-2 activation, suggesting antiangiogenesis effects in LicAtreated mice [87].

\section{Antioxidant Effects}

The anticancer effects of LicA have been at least partially attributed to its ability to act as an antioxidant. C57BL/6 mice with AOM/DSS-induced colon carcinogenesis were treated with LicA (5, 15, and $30 \mathrm{mg} / \mathrm{kg}$ body weight) [93]. Reduced colon tumor formation, decreased expression of PCNA (proliferating cell nuclear antigen), $\beta$-catenin, COX-2, and iNOS, and concomitant suppression of colon proinflammatory cytokines and chemokines were observed [93]. LicA-treated animals survived longer and LicA also inhibited liver metastasis induced by intrasplenic injection of BALB/c mice with CT-26 cells [93].

Results of one study suggested that administration of LicA might be beneficial in counteracting the side effects of cisplatin therapy in cancer patients. This idea was based on the finding that oral administration of LicA alone significantly suppressed the growth of solid tumors in CT-26 cell-inoculated Balb/c mice, without any obvious toxicity [94]. When combined with cisplatin, LicA inhibited cisplatin-induced kidney and liver damage [94]. The repeated oral administration of LicA prior to cisplatin treatment prevented cisplatinmediated increases in serum NO and the tissue lipid peroxidation levels, and restored the depleted reduced glutathione levels in tissues [94]. LicA suppressed both the generation of $\mathrm{NO}$ and prostaglandin $\mathrm{PGE}_{2}$ and also attenuated the expression of iNOS and COX-2 induced by lipopolysaccharide (LPS) in RAW264.7 cells [95]. LicA inhibited the LPSinduced production of inflammatory cytokines IL-1 and IL-6 in RAW264.7 cells and was able to protect BALB/c mice from LPS-induced endotoxin shock [95]. In another report, LicA $\left(\mathrm{IC}_{50} 15 \mathrm{nM}\right)$ inhibited $\mathrm{COX}-2$-mediated $\mathrm{PGE}_{2}$ production, but did not affect COX-1-mediated $\mathrm{PGE}_{2}$ production or IL-1 eta-induced production of IL-6 or -8 [96]. However, LicA $(1 \mu \mathrm{M})$ had no effect on $C O X-2$ mRNA or protein expression [96]. LicA inhibited human bladder cancer T24 proliferation in a concentration-dependent manner $\left(\mathrm{IC}_{50} \sim 55 \mu \mathrm{M}\right)$ [97]. Evidence suggested that LicA inhibited proliferation by increasing the levels of intracellular ROS because the affects could be attenuated by treatment with the glutathione precursor and antioxidant, $N$-acetyl-cysteine (NAC) [97]. These results agree with a previous report in which LicA inhibited proliferation, increased levels of ROS, and induced apoptosis in T24 cells [98]. This compound also induced mitochondrial dysfunction, caspase- 3 activation, and PARP cleavage and caused endoplasmic reticulum (ER) stress-dependent apoptosis [98].

Several studies indicate that LicA can suppress NF-kB transactivation. NF- $\mathrm{KB}$ is a rapidly induced stress-responsive transcription factor that functions to intensify the transcription 
of a variety of genes including cytokines, growth factors and acute response proteins [99] and its activation is also linked to the mitogen-activation protein kinase (MAPK) signaling pathways [100]. In its inactive form, NF- $\mathrm{kB}$ is found in the cytosol bound to an inhibitory protein called inhibitory kappa $\mathrm{B}$ (I- $-\mathrm{B})$. When stimulated, I- $\mathrm{kB}$ is phosphorylated by an I-kB kinase, released from NF-KB, and subsequently degraded. Following separation from I- $\mathrm{KB}, \mathrm{NF}-\mathrm{kB}$ is translocated into the nucleus where it activates gene transcription by binding to its specific DNA sequence found in certain genes. Importantly, NF-B activation is associated with initiation or acceleration of carcinogenesis [101], and in JB6 Cl41 mouse epidermal skin cells, inhibition of NF-KB also blocked tumor promoterinduced cell transformation [102]. NF-KB comprises five family members [103] including the three NF- $\mathrm{KB} / \mathrm{Rel}$ proteins, RelA (p65), RelB, and c-Rel, which share structural homology with the retroviral oncoprotein v-Rel [104].

LicA was reported to inhibit LPS-induced NF- $\mathrm{kB}$ transcriptional activation but had no effect on either the phosphorylation and degradation of I $\mathrm{KB}$ or nuclear translocation and DNA binding activity of NF-kB p65 [105]. However, LicA suppressed the phosphorylation of p65 (Ser276), which attenuated NF- $\mathrm{KB}$ transactivation by preventing the interaction of p65 with $\mathrm{p} 300$, a transcriptional co-activating protein [105]. This group further observed that LicB and LicD inhibited LPS-induced phosphorylation of p65 (Ser276) and transcriptional activation of NF-KB [106]. Each of the three licorice components attenuated the LPS-induced activation of protein kinase A [106], which is required for p65 (Ser276) phosphorylation. This effect was associated with decreased production of NO, TNF $\alpha$ and MCP-1 (monocyte chemoattractant protein-1) [106], a key chemokine that regulates migration and infiltration of monocytes or macrophages.

Others showed that LicA could attenuate TNF $\alpha$-induced nuclear localization, DNA binding activity, and the transcriptional activity of NF- $\mathrm{KB}$ but had no effect on TNF $\alpha$ recruitment of receptor-interacting protein 1 or I- $\mathrm{KB}$ kinase $\beta$ to the TNF receptor I [107]. However, LicA inhibited TNF $\alpha$ induced I-kB kinase $\beta$ complex (IKK) activation apparently by interacting with Cys 179 of I-kB kinase I- $\mathrm{kB}$ kinase $\beta$. This group also reported that LicA attenuated $\mathrm{TNF} \alpha$-induced expression of inflammatory cytokines CCL2/monocyte chemotactic protein-1 and CXCL1/KC [107].

LicA also was reported to inhibit the migration and invasion capabilities of SCC- 25 oral cancer cells by suppressing the activity and protein level of MMP-2 and increasing the level of tissue inhibitor of metallopeptidases (TIMP)-2 [108]. Decreased expression of phosphorylated p65 (a component of $\mathrm{NF}-\mathrm{KB}$ ) and N-cadherin (mesenchymal marker) and increased E-cadherin (epithelial marker) were also observed in LicAtreated SCC-25 cells [108]. LicA also attenuated the migratory and invasion ability of SK-Hep-1 and HA22T/VGH hepatic cancer cells [109]. This effect corresponded with an inhibition of the activity and expression of the serine protease, urokinase plasminogen activator (uPA), and decreased phosphorylation of JNKs and MKK4 in SK-Hep-1 cells [109]. Additionally, LicA suppressed the expression of NF- $\mathrm{KB}$ and its binding to the $u P A$ promoter [109]. Others reported that LicA effectively inhibited migration and invasion of A549 and H460 lung cancer cells [110]. The compound also significantly inhibited the protein and gene expression of MMP-1 and MMP-3 in A549 cells. Treatment of A549 cells with LicA suppressed phosphorylation of Akt, an effect, along with inhibition of migration and invasion, that was reversed by treatment with LY294002 (PI3-K inhibitor) or transfection with constitutive active-Akt i [110].

\section{Other Mechanisms}

JNKs play an important role in many physiological processes induced by numerous stress signals. Each JNK protein appears to have a distinct function in cancer, diabetes, or Parkinson's disease. We hypothesized that JNKs might be potential target proteins of LicA. Using a pull-down assay with licochalcone $\mathrm{A}$ conjugated with $\mathrm{CNBr}$-Sepharose $4 \mathrm{~B}$ beads, we found that LicA could directly bind with either JNK1 or JNK2, but not with p38 or ERKs, in murine embryonic fibroblasts (MEFs) [111••]. However, results of an in vitro kinase assay indicated that LicA mainly inhibited JNK1 activity $(\sim 55 \%)$ with only a small effect $(7 \%)$ on JNK2 kinase activity, which suggested that LicA might be a selective inhibitor of JNK1 [111••]. We previously reported AV-7 as a selective JNK1 inhibitor designed to interrupt the protein-protein interaction between JNK1 and its binding partner, JNK-interacting protein (JIP) [112]. Thus, we hypothesized that LicA might bind at the JNK1-JIP site rather than the ATP pocket to selectively inhibit JNK1 kinase activity. We found that LicA disrupted the interaction between JNK1 and JIP1, but could only slightly affect the interaction between JNK2 and JIP1 [111••]. To understand why the interaction was so different between LicA and the two very similar JNK proteins, we developed computational docking models. The results indicated that LicA binds at the JIP1 binding pocket of either JNK1 or JNK2 [111••]. However, a molecular dynamics simulation suggested that LicA more dramatically distorted the ATP binding pocket of JNK1 compared to JNK2, which resulted in a greater reduction in the affinity of JNK1 for ATP binding compared to the affinity of JNK2 for ATP [111••]. Exposure to ultraviolet B irradiation is known to stimulate JNKs activation. Additional experiments indicated that LicA markedly suppressed UVB-induced c-Jun phosphorylation and total c-Jun protein levels in a dose-dependent manner in JNK2 ${ }^{-/-}$MEFs, which only express JNK1 [111••]. Both UVB-induced c-Jun phosphorylation and total c-Jun protein expression were partially suppressed by LicA treatment in $\mathrm{JNK}^{\mathrm{wt}}$ MEFs, which express both JNK1 and JNK2 [111••]. 
However, neither UV-induced c-Jun phosphorylation nor total c-Jun protein level was affected by LicA in $\mathrm{JNK}^{-/-} \mathrm{MEFs}$, which only express $\mathrm{JNK} 2$. We treated $\mathrm{JNK}^{\mathrm{wt}}, \mathrm{JNK}^{-/-}$, and $\mathrm{JNK}^{-/-}$mice with UVB with or without topical application of LicA and harvested dorsal skin samples $24 \mathrm{~h}$ later. LicA effectively inhibited c-Jun (Ser73) phosphorylation and decreased total c-Jun level most effectively in $\mathrm{JNK}^{-1-}$ mouse skin, which only expresses JNK1. We also found that LicA significantly inhibited AP-1 transcriptional activity and induced p53 and p21 expression and induced G1 cell cycle phase arrest and apoptosis in HCT116 colon cancer cells. Importantly, LicA reduced HCT116 colon cancer xenograft growth in vivo. The reduction corresponded with marked decreases in the expression of Ki-67, a cell proliferation marker, and phosphorylation of c-Jun in licochalcone Atreated tumors compared to controls $[111 \bullet \bullet]$.

\section{Licochalcone E}

Besides LicA, LicE is another licochalcone isolated from licorice that appears to affect cell cycle and induce apoptosis. LicE suppressed ECV304 bladder epithelial-like cell growth and induced apoptosis [113]. Similarly to LicA, LicE inhibited activation of NF- $\mathrm{KB}$ and was associated with a change in the $\mathrm{Bax} / \mathrm{Bcl}-2$ ratio that favored apoptosis [113]. BALB/c mice were injected with 4T1 mammary carcinoma cells into the mammary fat pads and then 7 days later, were administered LicE (7 or $14 \mathrm{mg} / \mathrm{kg} \mathrm{BW/day)} \mathrm{orally} \mathrm{for} 25$ days [114]. Mice treated with LicE exhibited reduced solid tumor growth and lung metastasis with no apparent toxicity. Tumors isolated from mice treated with LicE exhibited reduced expression of Ki67 and various cyclins and cyclin-dependent kinases and increased expression of Bax and cleaved caspase- 3 but decreased Bcl-2 expression, suggesting an induction of apoptosis [114]. LicE also had inhibitory effects on a number of factors important in tumor angiogenesis (e.g., VEGF-A and C and VEGF receptor-2) and effectively decreased cell migration and invasion in cell culture studies [114].

Similar to other licorice compounds, LicE exerts antioxidant effects. For example, topical application of LicE (0.5-2 mg) effectively inhibited TPA-induced ear edema formation in mice. This was associated with decreased phosphorylation of JNKs, c-Jun, and ERKs [115]. LicE treatment reduced expression of iNOS and COX-2 proteins in mouse skin. In RAW 64.7 cells treated with LPS, LicE $(2.5-7.5 \mu \mathrm{M})$ treatment decreased release of $\mathrm{NO}$ and $\mathrm{PE}_{2}$, lowered expression of IL-6, IL-1, and TNF $\alpha$ and decreased activation of Akt, p38, JNKs, and c-Jun [116]. These effects coincided with decreased $i N O S$ and $C O X-2$ promoter activity and reductions in their gene and protein expression levels. Notably, similar to other licochalcones, LicE treatment reduced degradation of $\operatorname{I} \kappa \alpha \mathrm{B} \alpha$, translocation of p65 to the nucleus and transcriptional activity of $N F-\kappa B$ as well as attenuated the transcriptional activity of activator protein (AP)-1 [116]. Furthermore, in RAW264.7 macrophage cells, LicE also inhibited LPS-stimulated production of IL-12p40, a common subunit of IL-12 and IL-23. The inhibition appeared to be associated with decreased binding of $\mathrm{NF}-\mathrm{KB}$ to the promoter region of IL-12 [117]. LicE was also reported to be a potential activator of the transcription factor nuclear factor-E2related factor-2 (Nrf2)/ antioxidant response element (ARE)-dependent pathway [115].

\section{Conclusions}

Several lines of evidence suggest that many cancers are preventable and a great deal of interest has focused on diet and food supplements as prevention strategies. However, one of the greatest challenges is to decipher the distortion and halftruths reported in the popular media regarding the health benefits of certain foods or food supplements. The use of these substances is not new, but interest has increased dramatically because of perceived health benefits presumably acquired without unpleasant side effects. However, the effects of long-term consumption of food supplements and specific dietary factors have not been extensively studied. Licorice and its extracts have been used in traditional and herbal medicines for thousands of years. However, some components of licorice exert unwanted side effects and therefore identifying safer licorice components is desirable. Literature has suggested that the anticancer activities of many of the licorice components appear to include cell cycle arrest, apoptosis induction, and general antioxidant effects involving a variety of proteins at least indirectly, including many cell cycle-related proteins, apoptosis-associated proteins, MMP proteins, COX-2, GSK- $\beta$, Akt, NF- $\kappa$ B, and MAP kinases. New computational and screening technologies have led to the identification of direct licorice binding partners, including PI3-K, MKK4, MKK7, JNK1, mTOR, and Cdk2, resulting in decreased carcinogenesis in several cell and mouse models with no obvious toxicity. These are the types of studies that are needed in order to move the field of the health benefits of natural compound science forward.

\section{Compliance with Ethics Guidelines}

Conflict of Interest Ann M. Bode and Zigang Dong declare that they have no conflict of interest.

Human and Animal Rights and Informed Consent This article does not contain any studies with human or animal subjects performed by any of the authors. 


\section{References}

Papers of particular interest, published recently, have been highlighted as:

- Of importance

- Of major importance

1. Shibata S. A drug over the millennia: pharmacognosy, chemistry, and pharmacology of licorice. Yakugaku Zasshi. 2000;120(10): 849-62.

2. Gibson MR. Glycyrrhiza in old and new perspectives. Lloydia. 1978;41(4):348-54.

3. GRAS status of licorice (Glycyrrhiza), ammoniated glycyrrhizin, and monoammonium glycyrrhizinate, Fed Reg 50 (1985).

4. Johns C. Glycyrrhizic acid toxicity caused by consumption of licorice candy cigars. Cjem. 2009;11(1):94-6.

5. Spinks EA, Fenwick GR. The determination of glycyrrhizin in selected UK liquorice product. Food Addit Contam. 1990;7(6): 769-78.

6. Ibsen KK. Liquorice consumption and its influence on blood pressure in Danish school-children. Dan Med Bull. 1981;28(3):124-6.

7. Gomez-Sanchez EP, Gomez-Sanchez CE. Central hypertensinogenic effects of glycyrrhizic acid and carbenoxolone. Am J Physiol. 1992;263(6 Pt 1):E1125-30.

8. Sigurjonsdottir HA, Franzson L, Manhem K, Ragnarsson J, Sigurdsson G, Wallerstedt S. Liquorice-induced rise in blood pressure: a linear dose-response relationship. J Hum Hypertens. 2001;15(8):549-52.

9. Fukai T, Marumo A, Kaitou K, Kanda T, Terada S, Nomura T. Anti-Helicobacter pylori flavonoids from licorice extract. Life Sci. 2002;71(12):1449-63.

10. Wang ZY, Nixon DW. Licorice and cancer. Nutr Cancer. 2001;39(1):1-11.

11. Fuhrman B, Volkova N, Kaplan M, Presser D, Attias J, Hayek T, et al. Antiatherosclerotic effects of licorice extract supplementation on hypercholesterolemic patients: increased resistance of LDL to atherogenic modifications, reduced plasma lipid levels, and decreased systolic blood pressure. Nutrition. 2002;18(3):26873.

12. Asl MN, Hosseinzadeh H. Review of pharmacological effects of Glycyrrhiza sp. and its bioactive compounds. Phytother Res. 2008;22(6):709-24.

13. Draelos ZD. Skin lightening preparations and the hydroquinone controversy. Dermatol Ther. 2007;20(5):308-13.

14. Kusano A, Nikaido T, Kuge T, Ohmoto T, Delle Monache G, Botta B, et al. Inhibition of adenosine 3',5'-cyclic monophosphate phosphodiesterase by flavonoids from licorice roots and 4arylcoumarins. Chem Pharm Bull. 1991;39(4):930-3.

15. Vaya J, Belinky PA, Aviram M. Antioxidant constituents from licorice roots: isolation, structure elucidation and antioxidative capacity toward LDL oxidation. Free Radic Biol Med. 1997;23(2):302-13.

16. Hatano T, Shintani Y, Aga Y, Shiota S, Tsuchiya T, Yoshida T. Phenolic constituents of licorice. VIII. Structures of glicophenone and glicoisoflavanone, and effects of licorice phenolics on methicillin-resistant Staphylococcus aureus. Chem Pharm Bull. 2000;48(9):1286-92.

17. Nomura T, Fukai T. Phenolic constituents of licorice (Glycyrrhiza species). Fortschr Chem Org Naturst. 1998;73:1-158.

18. Hattori M, Sakamoto T, Yamagishi T, Sakamoto K, Konishi K, Kobashi K, et al. Metabolism of glycyrrhizin by human intestinal flora. II. Isolation and characterization of human intestinal bacteria capable of metabolizing glycyrrhizin and related compounds. Chem Pharm Bull. 1985;33(1):210-7.
19. Stewart PM, Wallace AM, Valentino R, Burt D, Shackleton CH, Edwards CR. Mineralocorticoid activity of liquorice: 11-betahydroxysteroid dehydrogenase deficiency comes of age. Lancet. 1987;2(8563):821-4.

20. Whorwood CB, Sheppard MC, Stewart PM. Licorice inhibits 11 beta-hydroxysteroid dehydrogenase messenger ribonucleic acid levels and potentiates glucocorticoid hormone action. Endocrinology. 1993;132(6):2287-92.

21. van Rossum TG, Vulto AG, Hop WC, Brouwer JT, Niesters HG, Schalm SW. Intravenous glycyrrhizin for the treatment of chronic hepatitis $\mathrm{C}$ : a double-blind, randomized, placebocontrolled phase I/II trial. J Gastroenterol Hepatol. 1999;14(11):1093-9.

22. Curreli F, Friedman-Kien AE, Flore O. Glycyrrhizic acid alters Kaposi sarcoma-associated herpesvirus latency, triggering p53mediated apoptosis in transformed B lymphocytes. J Clin Invest. 2005;115(3):642-52.

23. Cinatl J, Morgenstern B, Bauer G, Chandra P, Rabenau H, Doerr HW. Glycyrrhizin, an active component of liquorice roots, and replication of SARS-associated coronavirus. Lancet. 2003;361(9374):2045-6.

24. Hoever G, Baltina L, Michaelis M, Kondratenko R, Tolstikov GA, Doerr HW, et al. Antiviral activity of glycyrrhizic acid derivatives against SARS-coronavirus. J Med Chem. 2005;48(4):1256-9.

25. Lee CH, Park SW, Kim YS, Kang SS, Kim JA, Lee SH, et al. Protective mechanism of glycyrrhizin on acute liver injury induced by carbon tetrachloride in mice. Biol Pharm Bull. 2007;30(10):1898-904.

26. Abe M, Akbar F, Hasebe A, Horiike N, Onji M. Glycyrrhizin enhances interleukin-10 production by liver dendritic cells in mice with hepatitis. J Gastroenterol. 2003;38(10):962-7.

27. Gumpricht E, Dahl R, Devereaux MW, Sokol RJ. Licorice compounds glycyrrhizin and 18beta-glycyrrhetinic acid are potent modulators of bile acid-induced cytotoxicity in rat hepatocytes. $\mathrm{J}$ Biol Chem. 2005;280(11):10556-63.

28. Shinada M, Azuma M, Kawai H, Sazaki K, Yoshida I, Yoshida T, et al. Enhancement of interferon-gamma production in glycyrrhizin-treated human peripheral lymphocytes in response to concanavalin A and to surface antigen of hepatitis B virus. Proc Soc Exp Biol Med. 1986;181(2):205-10.

29. Cherng JM, Lin HJ, Hung MS, Lin YR, Chan MH, Lin JC. Inhibition of nuclear factor kappaB is associated with neuroprotective effects of glycyrrhizic acid on glutamate-induced excitotoxicity in primary neurons. Eur J Pharmacol. 2006;547(1-3):10-21.

30. Agarwal R, Wang ZY, Mukhtar H. Inhibition of mouse skin tumorinitiating activity of DMBA by chronic oral feeding of glycyrrhizin in drinking water. Nutr Cancer. 1991;15(3-4):187-93.

31. Aydemir EA, Oz ES, Gokturk RS, Ozkan G, Fiskin K. Glycyrrhiza flavescens subsp. antalyensis exerts antiproliferative effects on melanoma cells via altering TNF-alpha and IFN-alpha levels. Food Chem Toxicol. 2011;49(4):820-8.

32. Kobayashi M, Fujita K, Katakura T, Utsunomiya T, Pollard RB, Suzuki F. Inhibitory effect of glycyrrhizin on experimental pulmonary metastasis in mice inoculated with B16 melanoma. Anticancer Res. 2002;22(6C):4053-8.

33. Cherng JM, Tsai KD, Yu YW, Lin JC. Molecular mechanisms underlying chemopreventive activities of glycyrrhizic acid against UVB-radiation-induced carcinogenesis in SKH-1 hairless mouse epidermis. Radiat Res. 2011;176(2):177-86. PubMed PMID: 21545294. Epub 2011/05/07. Eng. This group showed that ingesting orally licorice compounds could prevent UVB induced skin cancer, making it more applicable to the human condition.

34. Thirugnanam S, Xu L, Ramaswamy K, Gnanasekar M. Glycyrrhizin induces apoptosis in prostate cancer cell lines DU145 and LNCaP. Oncol Rep. 2008;20(6):1387-92. 
35. Hibasami H, Iwase H, Yoshioka K, Takahashi H. Glycyrrhizin induces apoptosis in human stomach cancer KATO III and human promyelotic leukemia HL-60 cells. Int J Mol Med. 2005;16(2):233-6.

36. Hibasami H, Iwase H, Yoshioka K, Takahashi H. Glycyrrhetic acid (a metabolic substance and aglycon of glycyrrhizin) induces apoptosis in human hepatoma, promyelotic leukemia and stomach cancer cells. Int J Mol Med. 2006;17(2):215-9.

37. Lin D, Zhong W, Li J, Zhang B, Song G, Hu T. Involvement of BID translocation in glycyrrhetinic acid and 11-deoxy glycyrrhetinic acid-induced attenuation of gastric cancer growth. Nutr Cancer. 2014;66(3):463-73.

38. Niwa K, Lian Z, Onogi K, Yun W, Tang L, Mori H, et al. Preventive effects of glycyrrhizin on estrogen-related endometrial carcinogenesis in mice. Oncol Rep. 2007;17(3):617-22.

39. Wang D, Wong HK, Feng YB, Zhang ZJ. 18beta-glycyrrhetinic acid induces apoptosis in pituitary adenoma cells via ROS/ MAPKs-mediated pathway. J Neurooncol. 2014;116(2):221-30.

40. Rossi T, Castelli M, Zandomeneghi G, Ruberto A, Benassi L, Magnoni C, et al. Selectivity of action of glycyrrhizin derivatives on the growth of MCF-7 and HEP-2 cells. Anticancer Res. 2003;23(5A):3813-8.

41. Huang RY, Chu YL, Huang QC, Chen XM, Jiang ZB, Zhang X, et al. 18beta-Glycyrrhetinic acid suppresses cell proliferation through inhibiting thromboxane synthase in non-small cell lung cancer. PLoS ONE. 2014;9(4):e93690.

42. Song J, Ko HS, Sohn EJ, Kim B, Kim JH, Kim HJ, et al. Inhibition of protein kinase $\mathrm{C}$ alpha/betaII and activation of c-Jun NH2terminal kinase mediate glycyrrhetinic acid induced apoptosis in non-small cell lung cancer NCI-H460 cells. Bioorg Med Chem Lett. 2014;24(4):1188-91.

43. Khan R, Khan AQ, Lateef A, Rehman MU, Tahir M, Ali F, et al. Glycyrrhizic acid suppresses the development of precancerous lesions via regulating the hyperproliferation, inflammation, angiogenesis and apoptosis in the colon of Wistar rats. PLoS ONE. 2013;8(2):e56020.

44. Chueh FS, Hsiao YT, Chang SJ, Wu PP, Yang JS, Lin JJ, et al. Glycyrrhizic acid induces apoptosis in WEHI-3 mouse leukemia cells through the caspase- and mitochondria-dependent pathways. Oncol Rep. 2012;28(6):2069-76.

45. Shintani S, Murase H, Tsukagoshi H, Shiigai T. Glycyrrhizin (licorice)-induced hypokalemic myopathy. Report of 2 cases and review of the literature. Eur Neurol. 1992;32(1):44-51.

46. Elinav E, Chajek-Shaul T. Licorice consumption causing severe hypokalemic paralysis. Mayo Clin Proc. 2003;78(6):767-8.

47. Mumoli N, Cei M. Licorice-induced hypokalemia. Int J Cardiol. 2008;124(3):e42-4.

48. Celik MM, Karakus A, Zeren C, Demir M, Bayarogullari H, Duru $\mathrm{M}$, et al. Licorice induced hypokalemia, edema, and thrombocytopenia. Hum Exp Toxicol. 2012;31(12):1295-8.

49. Final report on the safety assessment of glycyrrhetinic acid, potassium glycyrrhetinate, disodium succinoyl glycyrrhetinate, glyceryl glycyrrhetinate, glycyrrhetinyl stearate, stearyl glycyrrhetinate, glycyrrhizic acid, ammonium glycyrrhizate, dipotassium glycyrrhizate, disodium glycyrrhizate, trisodium glycyrrhizate, methyl glycyrrhizate, and potassium glycyrrhizinate. Int J Toxicol. 2007;26 Suppl 2:79-112. PubMed PMID: 17613133. Epub 2007/12/06. eng.

50. Cuendet M, Guo J, Luo Y, Chen S, Oteham CP, Moon RC, et al. Cancer chemopreventive activity and metabolism of isoliquiritigenin, a compound found in licorice. Cancer Prev Res (Phila). 2010;3(2):221-32.

51. Ye L, Gho WM, Chan FL, Chen S, Leung LK. Dietary administration of the licorice flavonoid isoliquiritigenin deters the growth of MCF-7 cells overexpressing aromatase. Int J Cancer. 2009;124(5):1028-36.
52. Vandooren J, Van den Steen PE, Opdenakker G. Biochemistry and molecular biology of gelatinase B or matrix metalloproteinase-9 (MMP-9): the next decade. Crit Rev Biochem Mol Biol. 2013;48(3):222-72.

53. Ma J, Zhang Q, Chen S, Fang B, Yang Q, Chen C, et al. Mitochondrial dysfunction promotes breast cancer cell migration and invasion through HIF1alpha accumulation via increased production of reactive oxygen species. PLoS ONE. 2013;8(7): e69485.

54. Cao Y. VEGF-targeted cancer therapeutics-paradoxical effects in endocrine organs. Nat Rev Endocrinol. 2014;10(9):530-9.

55. Kessenbrock K, Plaks V, Werb Z. Matrix metalloproteinases: regulators of the tumor microenvironment. Cell. 2010;141(1): 52-67.

56. Lorusso V, Marech I. Novel plant-derived target drugs: a step forward from licorice? Expert Opin Ther Targets. 2013;17(4):333-5.

57. Kang SW, Choi JS, Choi YJ, Bae JY, Li J, Kim DS, et al. Licorice isoliquiritigenin dampens angiogenic activity via inhibition of MAPK-responsive signaling pathways leading to induction of matrix metalloproteinases. J Nutr Biochem. 2010;21(1):55-65.

58. Lau GT, Ye L, Leung LK. The licorice flavonoid isoliquiritigenin suppresses phorbol ester-induced cyclooxygenase-2 expression in the non-tumorigenic MCF-10A breast cell line. Planta Med. 2010;76(8):780-5.

59. Takahashi T, Takasuka N, Iigo M, Baba M, Nishino H, Tsuda H, et al. Isoliquiritigenin, a flavonoid from licorice, reduces prostaglandin E2 and nitric oxide, causes apoptosis, and suppresses aberrant crypt foci development. Cancer Sci. 2004;95(5):448-53.

60. Zhao H, Zhang X, Chen X, Li Y, Ke Z, Tang T, et al. Isoliquiritigenin, a flavonoid from licorice, blocks M2 macrophage polarization in colitis-associated tumorigenesis through downregulating PGE2 and IL-6. Toxicol Appl Pharmacol. 2014;279(3):311-21. PubMed PMID: 25026504. Epub 2014/07/ 16. Eng. Showed different, interesting mechanism for the anticancer activity of iso-liquiritigenin.

61. Zhou Y, Ho WS. Combination of liquiritin, isoliquiritin and isoliquirigenin induce apoptotic cell death through upregulating p53 and p21 in the A549 non-small cell lung cancer cells. Oncol Rep. 2014;31(1):298-304.

62. Hsu YL, Chia CC, Chen PJ, Huang SE, Huang SC, Kuo PL. Shallot and licorice constituent isoliquiritigenin arrests cell cycle progression and induces apoptosis through the induction of ATM/ p53 and initiation of the mitochondrial system in human cervical carcinoma HeLa cells. Mol Nutr Food Res. 2009;53(7):826-35.

63. Zheng H, Li Y, Wang Y, Zhao H, Zhang J, Chai H, et al. Downregulation of COX-2 and CYP 4A signaling by isoliquiritigenin inhibits human breast cancer metastasis through preventing anoikis resistance, migration and invasion. Toxicol Appl Pharmacol. 2014;280(1):10-20. PubMed PMID: 25094029. Epub 2014/08/06. Eng. This paper present a unique mechansim for the anticancer activity of iso-liquiritigenin.

64. Frisch SM, Francis H. Disruption of epithelial cell-matrix interactions induces apoptosis. J Cell Biol. 1994;124(4):619-26.

65. Simpson CD, Anyiwe K, Schimmer AD. Anoikis resistance and tumor metastasis. Cancer Lett. 2008;272(2):177-85.

66. Malumbres M, Barbacid M. Cell cycle, CDKs and cancer: a changing paradigm. Nat Rev Cancer. 2009;9(3):153-66.

67. Sherr CJ. D-type cyclins. Trends Biochem Sci. 1995;20(5):18790.

68. Sherr CJ. Cancer cell cycles. Science. 1996;274(5293):1672-7.

69. Huang W, Tang S, Qiao X, Ma W, Ji S, Wang K, et al. Isoangustone A induces apoptosis in SW480 human colorectal adenocarcinoma cells by disrupting mitochondrial functions. Fitoterapia. 2014;94:36-47.

70. Seon MR, Lim SS, Choi HJ, Park SY, Cho HJ, Kim JK, et al. Isoangustone A present in hexane/ethanol extract of Glycyrrhiza 
uralensis induces apoptosis in DU145 human prostate cancer cells via the activation of DR4 and intrinsic apoptosis pathway. Mol Nutr Food Res. 2010;54(9):1329-39.

71. Seon MR, Park SY, Kwon SJ, Lim SS, Choi HJ, Park H, et al. Hexane/ethanol extract of Glycyrrhiza uralensis and its active compound isoangustone A induce G1 cycle arrest in DU145 human prostate and 4T1 murine mammary cancer cells. J Nutr Biochem. 2012;23(1):85-92.

72.• Lee E, Son JE, Byun S, Lee SJ, Kim YA, Liu K, et al. CDK2 and mTOR are direct molecular targets of isoangustone $\mathrm{A}$ in the suppression of human prostate cancer cell growth. Toxicol Appl Pharmacol. 2013;272(1):12-20. PubMed PMID: 23707764. Epub 2013/05/28. eng. One of the first papers to show direct molecular targets of a specific licorice compound.

73. Vlach J, Hennecke S, Amati B. Phosphorylation-dependent degradation of the cyclin-dependent kinase inhibitor p27. EMBO J. 1997;16(17):5334-44.

74. Liang J, Zubovitz J, Petrocelli T, Kotchetkov R, Connor MK, Han $\mathrm{K}$, et al. PKB/Akt phosphorylates p27, impairs nuclear import of p27 and opposes p27-mediated G1 arrest. Nat Med. 2002;8(10): 1153-60.

75. Tsihlias J, Kapusta LR, DeBoer G, Morava-Protzner I, Zbieranowski I, Bhattacharya N, et al. Loss of cyclin-dependent kinase inhibitor p27Kip1 is a novel prognostic factor in localized human prostate adenocarcinoma. Cancer Res. 1998;58(3):542-8.

76. Yang RM, Naitoh J, Murphy M, Wang HJ, Phillipson J, de Kernion JB, et al. Low p27 expression predicts poor disease-free survival in patients with prostate cancer. J Urol. 1998;159(3):9415 .

77.• Song NR, Lee EJ, Byun S, Kim JE, Mottamal M, Park JH, et al. Isoangustone A, a novel licorice compound, inhibits cell proliferation by targeting PI3-K, MKK4 and MKK7 in human melanoma. Cancer Prev Res (Phila). Oct 8. PubMed PMID: 24104352. Epub 2013/10/10. Eng. One of the first papers to report direct binding partners for a specific licorice compound.

78. Musgrove EA, Caldon CE, Barraclough J, Stone A, Sutherland RL. Cyclin D as a therapeutic target in cancer. Nat Rev Cancer. 2011;11(8):558-72.

79. Curtin JA, Fridlyand J, Kageshita T, Patel HN, Busam KJ, Kutzner $\mathrm{H}$, et al. Distinct sets of genetic alterations in melanoma. N Engl J Med. 2005;353(20):2135-47.

80. Willems L, Tamburini J, Chapuis N, Lacombe C, Mayeux P, Bouscary D. PI3K and mTOR signaling pathways in cancer: new data on targeted therapies. Curr Oncol Rep. 2012;14(2): 129-38.

81. Yuan TL, Cantley LC. PI3K pathway alterations in cancer: variations on a theme. Oncogene. 2008;27(41):5497-510.

82. Takahashi-Yanaga F, Sasaguri T. GSK-3beta regulates cyclin D1 expression: a new target for chemotherapy. Cell Signal. 2008;20(4):581-9.

83. Xiao XY, Hao M, Yang XY, Ba Q, Li M, Ni SJ, et al. Licochalcone A inhibits growth of gastric cancer cells by arresting cell cycle progression and inducing apoptosis. Cancer Lett. 2011;302(1):6975.

84. Rafi MM, Rosen RT, Vassil A, Ho CT, Zhang H, Ghai G, et al. Modulation of bcl-2 and cytotoxicity by licochalcone-A, a novel estrogenic flavonoid. Anticancer Res. 2000;20(4):2653-8.

85. Kim YJ, Jung EB, Myung SC, Kim W, Lee CS. Licochalcone A enhances geldanamycin-induced apoptosis through reactive oxygen species-mediated caspase activation. Pharmacology. **;92(12):49-59. PubMed PMID: 23921841. Epub 2013/08/08. eng.

86. Lee CS, Kwak SW, Kim YJ, Lee SA, Park ES, Myung SC, et al. Guanylate cyclase activator YC-1 potentiates apoptotic effect of licochalcone A on human epithelial ovarian carcinoma cells via activation of death receptor and mitochondrial pathways. Eur J Pharmacol. 2012;683(1-3):54-62.
87. Kim YH, Shin EK, Kim DH, Lee HH, Park JH, Kim JK. Antiangiogenic effect of licochalcone A. Biochem Pharmacol. 2010;80(8):1152-9.

88. Park SY, Kim EJ, Choi HJ, Seon MR, Lim SS, Kang YH, et al. Anti-carcinogenic effects of non-polar components containing licochalcone A in roasted licorice root. Nutr Res Pract. 2014;8(3):257-66.

89. Yo YT, Shieh GS, Hsu KF, Wu CL, Shiau AL. Licorice and licochalcone-A induce autophagy in $\mathrm{LNCaP}$ prostate cancer cells by suppression of $\mathrm{Bcl}-2$ expression and the mTOR pathway. $\mathrm{J}$ Agric Food Chem. 2009;57(18):8266-73.

90. Kim JS, Park MR, Lee SY, Kim K, Moon SM, Kim CS, et al. Licochalcone A induces apoptosis in KB human oral cancer cells via a caspase-dependent FasL signaling pathway. Oncol Rep. 2014;31(2):755-62.

91. Fu Y, Hsieh TC, Guo J, Kunicki J, Lee MY, Darzynkiewicz Z, et al. Licochalcone-A, a novel flavonoid isolated from licorice root (Glycyrrhiza glabra), causes G2 and late-G1 arrests in androgenindependent PC-3 prostate cancer cells. Biochem Biophys Res Commun. 2004;322(1):263-70.

92. Park JH, Lim HJ, Lee KS, Lee S, Kwak HJ, Cha JH, et al. Antiproliferative effect of licochalcone A on vascular smooth muscle cells. Biol Pharm Bull. 2008;31(11):1996-2000.

93. Kim JK, Shin EK, Park JH, Kim YH. Antitumor and antimetastatic effects of licochalcone A in mouse models. J Mol Med (Berl). 2010;88(8):829-38.

94. Lee CK, Son SH, Park KK, Park JH, Lim SS, Kim SH, et al. Licochalcone A inhibits the growth of colon carcinoma and attenuates cisplatin-induced toxicity without a loss of chemotherapeutic efficacy in mice. Basic Clin Pharmacol Toxicol. 2008;103(1):48-54.

95. Kwon HS, Park JH, Kim DH, Kim YH, Shin HK, Kim JK. Licochalcone A isolated from licorice suppresses lipopolysaccharide-stimulated inflammatory reactions in RAW264.7 cells and endotoxin shock in mice. J Mol Med (Berl). 2008;86(11):1287-95.

96. Furuhashi I, Iwata $\mathrm{S}$, Shibata $\mathrm{S}$, Sato $\mathrm{T}$, Inoue H. Inhibition by licochalcone A, a novel flavonoid isolated from liquorice root, of IL-1beta-induced PGE2 production in human skin fibroblasts. J Pharm Pharmacol. 2005;57(12):1661-6.

97. Jiang J, Yuan X, Zhao H, Yan X, Sun X, Zheng Q. Licochalcone A inhibiting proliferation of bladder cancer T24 cells by inducing reactive oxygen species production. Biomed Mater Eng. 2014;24(1):1019-25.

98. Yuan X, Li D, Zhao H, Jiang J, Wang P, Ma X, et al. Licochalcone A-induced human bladder cancer T24 cells apoptosis triggered by mitochondria dysfunction and endoplasmic reticulum stress. Biomed Res Int. 2013;2013:474272.

99. Baldwin Jr AS. The NF-kappa B and I kappa B proteins: new discoveries and insights. Annu Rev Immunol. 1996;14: 649-83.

100. Schulze-Osthoff K, Ferrari D, Riehemann K, Wesselborg S. Regulation of NF-kappa B activation by MAP kinase cascades. Immunobiology. 1997;198(1-3):35-49.

101. Gilmore TD. Clinically relevant findings. J Clin Invest. 1997;100(12):2935-6.

102. Li JJ, Westergaard C, Ghosh P, Colburn NH. Inhibitors of both nuclear factor-kappaB and activator protein-1 activation block the neoplastic transformation response. Cancer Res. 1997;57(16): 3569-76.

103. Nabel GJ, Verma IM. Proposed NF-kappa B/I kappa B family nomenclature. Genes Dev. 1993;7(11):2063.

104. Gilmore TD. Introduction to NF-kappaB: players, pathways, perspectives. Oncogene. 2006;25(51):6680-4.

105. Furusawa J, Funakoshi-Tago M, Tago K, Mashino T, Inoue H, Sonoda Y, et al. Licochalcone A significantly suppresses LPS 
signaling pathway through the inhibition of NF-kappaB p65 phosphorylation at serine 276. Cell Signal. 2009;21(5):778-85.

106. Furusawa J, Funakoshi-Tago M, Mashino T, Tago K, Inoue H, Sonoda Y, et al. Glycyrrhiza inflata-derived chalcones, licochalcone A, licochalcone B and licochalcone D, inhibit phosphorylation of NF-kappaB p65 in LPS signaling pathway. Int Immunopharmacol. 2009;9(4):499-507.

107. Funakoshi-Tago M, Tanabe S, Tago K, Itoh H, Mashino T, Sonoda $\mathrm{Y}$, et al. Licochalcone A potently inhibits tumor necrosis factor alpha-induced nuclear factor-kappaB activation through the direct inhibition of IkappaB kinase complex activation. Mol Pharmacol. 2009;76(4):745-53.

108. Shen H, Zeng G, Tang G, Cai X, Bi L, Huang C, et al. Antimetastatic effects of licochalcone A on oral cancer via regulating metastasis-associated proteases. Tumour Biol. 2014;35(8): 7467-74.

109. Tsai JP, Hsiao PC, Yang SF, Hsieh SC, Bau DT, Ling CL, et al. Licochalcone A suppresses migration and invasion of human hepatocellular carcinoma cells through downregulation of MKK4/JNK via NF-kappaB mediated urokinase plasminogen activator expression. PLoS ONE. 2014;9(1):e86537.

110. Huang HC, Tsai LL, Tsai JP, Hsieh SC, Yang SF, Hsueh JT, et al. Licochalcone $\mathrm{A}$ inhibits the migration and invasion of human lung cancer cells via inactivation of the Akt signaling pathway with downregulation of MMP-1/-3 expression. Tumour Biol. 2014 Aug 23. PubMed PMID: 25149157. Epub 2014/08/26. Eng.

111.• Yao K, Chen H, Lee MH, Li H, Ma W, Peng C, et al. Licochalcone A, a natural inhibitor of c-Jun N-terminal kinase 1. Cancer Prev
Res (Phila). 2014;7(1):139-49. PubMed PMID: 24253317. Epub 2013/11/21. Eng. First to report that licochalcone A directly bound to and inhibited JNK1.

112. Yao K, Cho YY, Bode AM, Vummenthala A, Park JG, Liu K, et al. A selective small-molecule inhibitor of c-Jun N-terminal kinase 1. FEBS Lett. 2009;583(13):2208-12.

113. Chang HJ, Yoon G, Park JS, Kim MH, Baek MK, Kim NH, et al Induction of apoptosis by the licochalcone $\mathrm{E}$ in endothelial cells via modulation of NF-kappaB and Bcl-2 Family. Biol Pharm Bull. 2007;30(12):2290-3.

114. Kwon SJ, Park SY, Kwon GT, Lee KW, Kang YH, Choi MS, et al. Licochalcone E present in licorice suppresses lung metastasis in the 4T1 mammary orthotopic cancer model. Cancer Prev Res (Phila). 2013;6(6):603-13.

115. Kim SS, Lim J, Bang Y, Gal J, Lee SU, Cho YC, et al. Licochalcone E activates Nrf2/antioxidant response element signaling pathway in both neuronal and microglial cells: therapeutic relevance to neurodegenerative disease. J Nutr Biochem. 2012;23(10):1314-23.

116. Lee HN, Cho HJ, Lim Do Y, Kang YH, Lee KW, Park JH. Mechanisms by which licochalcone e exhibits potent antiinflammatory properties: studies with phorbol ester-treated mouse skin and lipopolysaccharide-stimulated murine macrophages. Int J Mol Sci. 2013;14(6):10926-43.

117. Cho YC, Lee SH, Yoon G, Kim HS, Na JY, Choi HJ, et al. Licochalcone $\mathrm{E}$ reduces chronic allergic contact dermatitis and inhibits IL-12p40 production through down-regulation of NFkappa B. Int Immunopharmacol. 2010;10(9):1119-26. 